\title{
Criminologie
}

\section{Les jeunes en bande et leurs parents. Les ressorts symboliques du détachement}

\section{The Gang Youths and Their Parents. Symbolic Patterns of Detachment \\ Los jóvenes en banda y sus padres. Los caracteres simbólicos del desapego}

\section{Marwan Mohammed}

Volume 48, numéro 2, automne 2015

Nouvelles perspectives sur le phénomène des gangs de rue

URI : https://id.erudit.org/iderudit/1033843ar

DOI : https://doi.org/10.7202/1033843ar

Aller au sommaire du numéro

Éditeur(s)

Les Presses de l’Université de Montréal

ISSN

0316-0041 (imprimé)

1492-1367 (numérique)

Découvrir la revue

Citer cet article

Mohammed, M. (2015). Les jeunes en bande et leurs parents. Les ressorts symboliques du détachement. Criminologie, 48(2), 187-207.

https://doi.org/10.7202/1033843ar

\section{Résumé de l'article}

L'analyse du rôle des familles dans la formation des bandes de jeunes est l'objet d'un certain paradoxe. Le fonctionnement intrafamilial est davantage commenté, déduit, que directement observé en situation. Paradoxe qui rejaillit sur les interventions institutionnelles de plus en plus intrusives et contraignantes. L'une des explications réside sans doute dans les choix méthodologiques. La prédominance des approches statistiques a tendance à occulter des processus microsociologiques nichés dans les plis intimes des relations et de la symbolique familiales qui nécessitent des dispositifs d'observation ancrés. C'est l'objet de cet article qui vise à mettre en perspective le poids des représentations et des jugements moraux réciproques entre les jeunes en bande et leurs parents. Des analyses qui proposent de remettre en question, voire de renouveler, la manière dont la recherche s'interroge sur les facteurs familiaux de la délinquance juvénile, en particulier le phénomène des bandes de jeunes, et les théorise. 


\title{
Les jeunes en bande et leurs parents
}

\section{Les ressorts symboliques du détachement}

\author{
Marwan Mohammed ${ }^{1}$ \\ Centre Maurice Halbwachs (CNRS, ENS, EHESS) \\ marwan.mohammed@ens.fr
}

RÉSUMÉ - L'analyse du rôle des familles dans la formation des bandes de jeunes est l'objet d'un certain paradoxe. Le fonctionnement intrafamilial est davantage commenté, déduit, que directement observé en situation. Paradoxe qui rejaillit sur les interventions institutionnelles de plus en plus intrusives et contraignantes. L'une des explications réside sans doute dans les choix méthodologiques. La prédominance des approches statistiques a tendance à occulter des processus microsociologiques nichés dans les plis intimes des relations et de la symbolique familiales qui nécessitent des dispositifs d'observation ancrés. C'est l'objet de cet article qui vise à mettre en perspective le poids des représentations et des jugements moraux réciproques entre les jeunes en bande et leurs parents. Des analyses qui proposent de remettre en question, voire de renouveler, la manière dont la recherche s'interroge sur les facteurs familiaux de la délinquance juvénile, en particulier le phénomène des bandes de jeunes, et les théorise.

MotS CLÉS • Délinquance, famille, autorité, bandes de jeunes, éducation.

En France, la responsabilité des familles est au cœur des discours publics et des programmes institutionnels de prévention et de répression de la délinquance ou du phénomène des bandes ${ }^{2}$. Ces dispositifs sont de plus en plus intrusifs dans les familles (Boucher, 2011; Gautron, 2014), ils

1. 48, boulevard Jourdan, 75014, Paris, France.

2. Nous définissons les bandes de jeunes comme des entités informelles, stables et durables qui se distinguent d'autres groupes de pairs par une dynamique transgressive et un rapport conflictuel avec leur environnement immédiat. Par dynamique transgressive, est pointé le fait que les inconduites sont fréquentes, qu'elles constituent un sujet de discussion et de valorisation et qu'elles participent de la cohésion collective. Mais si la dynamique est déviante, la finalité est sociale, c'est-à-dire qu'il s'agit avant tout de groupes de pairs. 
ambitionnent de réformer la trame des relations familiales et des pratiques parentales, en s'appuyant particulièrement sur la recherche universitaire. Or il nous semble que les nombreuses enquêtes qui tentent de cerner les mécanismes domestiques propices à la formation des bandes mettent rarement en question la grammaire symbolique de l'attachement et des relations familiales. Pourtant, la question des ressorts psychiques de la relation filiale a été largement traitée par la recherche criminologique, particulièrement d'inspiration psychologique et psychosociale, en mobilisant notamment cette notion d'attachement. Pour Hirschi (1969), celle-ci repose sur l'idée de «contrôle social informel», c'est-à-dire un lien social structurant favorisant le conformisme social. Au fil du temps, la dialectique attachement-détachement s'est déclinée de multiples manières en pointant l'épaisseur et l'intensité des échanges interpersonnels (Gove et Crutchfield, 1982), verticaux (filiation) ou horizontaux (fratrie ou conjugalité) (Cernkovich et Giordano, 1987), le rapport à l'espace domestique (dedans-dehors) et le rapport aux normes et aux règles (voir la synthèse de Smith et Stern, 1997). Svensson (2004) envisage quant à lui la notion d'attachement à travers la honte ressentie envers leurs parents, par des adolescents tentés de poser des actes délinquants. Nous voulons montrer ici que la dialectique attachement-détachement peut également être appréhendée au regard de la qualité de l'autorité éducative (emprise-déprise) ou du statut intrafamilial des enfants, en déplaçant la focale vers des enjeux plus symboliques, questionnant ainsi la manière dont les criminologies appréhendent le rôle des parents dans la délinquance.

L'étude essentiellement quantitative du lien entre famille et délinquance/bandes de jeunes privilégie une triple approche par les inégalités sociales, les pathologies et les schémas familiaux (Hébert et al., 1997; Mucchielli, 2000; Young, Fitzgibbon et Silverstone, 2014). La première insiste sur les inégalités multiples, cumulées et leurs effets sur les trajectoires déviantes, la seconde sur la reproduction intergénérationnelle de certaines pathologies sociales et psychologiques, la troisième sur les formes familiales et leurs effets (Rankinand et Wells, 1990).

Les méthodologies statistiques - prédominantes -, tout en apportant des éclairages indéniables, ont eu tendance à laisser dans l'ombre des processus nécessitant des dispositifs de recherche propices à une observation à la fois globale et fine des relations familiales. Les travaux de Jaggers et de son équipe (Jaggers et al., 2013) sont emblématiques d'une 
ambition récente et stimulante visant à actualiser l'étude approfondie des interactions familiales. Ils affirment que des niveaux plus élevés de «chaleur maternelle» ou «paternelle» sont associés à des niveaux inférieurs d'engagement dans des gangs, avec des effets différenciés selon le sexe. Toutefois, le choix d'une méthode quantitative «froide» tend à désincarner les sujets et leurs expériences, et du coup homogénéise de manière totalement artificielle des histoires humaines très différentes.

De fait, les ressorts familiaux des bandes juvéniles sont très souvent observés «par la fenêtre», à distance de l'espace domestique. Si le cadrage dominant de la recherche a jusqu'ici permis de repérer, puis d'articuler des variables clés telles que la position socioéconomique, la supervision, la qualité des scolarités, les formes familiales, la communication, les stratégies éducatives, les conditions de logement, etc., il n'a guère permis, à quelques exceptions près, le développement d'observations rapprochées de la trame psychosociale et symbolique des rapports familiaux (Bibeau et Perrault, 2003; Hamel et Brisebois, 2011; Mohammed, 2011). Or, nous souhaitons montrer ici qu'un tel schéma limite la compréhension et le renouvellement des analyses sur le lien entre famille et bande de jeunes. En puisant dans la sociologie ancrée des configurations et de la symbolique familiales (Lahire, 1995) et en prenant appui sur l'ethnométhodologie, notamment l'attachement de cette tradition théorique, au sens conféré par les individus à leurs expériences sociales les plus quotidiennes et intimes, notre objectif est d'arrimer certains enjeux symboliques, notamment le poids des tabous et des non-dits, à l'analyse plus classique du rôle des familles dans l'émergence des bandes de jeunes. Pour ce faire, on se focalisera dans cet article sur les problématiques croisées et dialectiques du statut symbolique des enfants et de celui de leurs parents, à travers l'analyse des formes de dépréciation qui structurent leurs relations et qui ont des effets concrets sur l'ordre familial et les comportements individuels. La notion de «cérémonies de dégradation» forgée par Garfinkel (1956), référence centrale de l'ethnométhodologie, est pour nous essentielle pour analyser les logiques de déclassement intrafamilial des jeunes en bande dont les parcours sont marqués par l'échec scolaire, ainsi que celles qui concourent à la perte d'autorité éducative des parents que la délinquance en bande révèle. Deux dynamiques dont la compréhension passe justement par un examen des représentations et des systèmes d'attente en mobilisant une méthodologie articulant observations dans 
la durée des relations intrafamiliales et des entretiens approfondis souvent répétés - avec les jeunes en bandes ainsi que leurs proches.

Nos analyses sont le fruit d'une longue enquête de type ethnographique sur les bandes de jeunes réalisée en deux moments. D'une part, une recherche doctorale en 2003-2007 basée sur des entretiens, des observations directes et l'étude de documents administratifs sur les enquêtés (dossiers judiciaires, scolaires, etc.) et leur environnement résidentiel. D'autre part, deux recherches sur les sorties de délinquance et l'implication des jeunes dans le trafic de drogue menées dans trois cités sensibles d'île-de-France et des Bouches-du-Rhône. La matière de nos analyses provient de ces différents terrains d'enquête - certains devant rester strictement anonymes - même si la majeure partie des données utilisées pour cet article proviennent de l'enquête principale qui s'est déroulée à Villiers-sur-Marne (94) au sein de la zone urbaine sensible (zus) des Hautes-Noues. Dans ce quartier du Val-de-Marne (94), nous avons interrogé 90 jeunes répartis dans plusieurs bandes distinctes et présentes sur le territoire depuis le milieu des années 1990. Nous avons réalisé cette recherche en occupant différentes positions (résident, animateur, éducateur sportif, intervenant associatif, employé du collège) qui donnent une tonalité particulière aux conditions d'enquête ${ }^{3}$. Cette présence durable et précoce dans les différentes structures d'accueil juvénile ainsi que dans la rue, nous a conduit à fréquenter certains enquêtés alors qu'ils étaient des enfants en bas âge, et nous a installé dans une relation de qualité avec les frères, amis et parents de la plupart d'entre eux. Un ensemble de présences dans le temps ayant notamment facilité l'observation d'interactions familiales.

\section{Le déclassement familial des enfants}

Le lien entre l'expérience scolaire et les formes de sociabilité a été souligné par de nombreuses enquêtes sur les bandes. Les parcours juvéniles sont aujourd'hui balisés par l'obligation de scolarité et la nécessité d'obtenir un diplôme, d'autant que les économies actuelles et de demain, largement mondialisées, exigent des niveaux de qualification toujours plus élevés. Le diplôme, même s'il ne garantit pas un emploi, augmente objectivement les chances d'en décrocher un et de limiter les risques de chômage. C'est particulièrement le cas dans les

3. Voir l'annexe de notre ouvrage sur les bandes (Mohammed, 2011). 
banlieues françaises (Observatoire national des zones urbaines sensibles [ONZUS], 2005). Cette structuration du devenir adulte exerce une forte contrainte sur l'éducation familiale dans les sociétés occidentales, malgré des différences de cadrage culturel et juridique selon les régions et les États (Van de Velde, 2008). En France, les enjeux scolaires et d'insertion professionnelle sont au cœur des attentes parentales quelle que soit leur condition sociale (Poullaouec, 2004). Dans les banlieues françaises où nous avons mené nos recherches, l'école est en outre associée à la prévention de la délinquance en raison de la pression négative, quotidienne, des franges déviantes de la rue. Enfin, la réussite scolaire participe de la promotion sociale et symbolique des familles inscrites localement dans des jeux de distinction.

\section{Mythes parentaux et relation filiale}

L'école est donc la principale voie de mobilité ascendante dont l'importance se comprend au regard des histoires familiales prises dans leur globalité, notamment celles issues de l'immigration (Vallet et Caille, 2000):

- Enquêteur: vous m'avez parlé «d'exemples de la cité», vous pouvez m'en dire plus?

- Mme R. (56 ans, 5 enfants, intérimaire dans les services d'aide à la personne): oui, c'est très simple, nous les mamans... les parents quoi! on compare souvent nos enfants... je sais pas si c'est bien de les comparer mais on les compare toujours... souvent en fait aux mêmes jeunes de la cité. C'est devenu des exemples en fait, des repères qu'on espère inculquer aux nôtres. Des jeunes d'ici qui ont bien réussi, avec la voie à montrer. On en parle souvent comme je t'ai dit... On en parle à nos enfants et entre adultes. (...) Les parents de ces jeunes ont grâce à ça du respect. Ils sont bien vus. (...) Leurs enfants font honneur au nom de famille.

La «cotation» symbolique et la «notabilisation» des parents dépendent ainsi en grande partie de la trajectoire scolaire et professionnelle des enfants. Au sein des familles, la comparaison des enfants qui «posent problème» avec des modèles de réussite est récurrente et vise à «motiver», «encourager», "faire prendre conscience» de la voie à suivre. Sans discuter ici de l'efficacité fort réduite d'une telle stratégie de mobilisation scolaire par la comparaison, ces pratiques expriment 
trois enjeux fondamentaux: l'intensité des attentes scolaires, la centralité des scolarités dans la constitution de l'ordre intrafamilial ainsi que les enjeux extrafamiliaux de distinction. Pour les familles engagées dans des sociabilités communautaires resserrées, ces enjeux prennent un sens particulier en raison de l'immédiateté de l'évaluation publique des réputations, contrairement à l'anonymat qu'offre une socialité moins dense. Plusieurs parents immigrés ont également fait part de critiques provenant de leurs communautés d'origine, qui portaient sur leur incapacité de mettre à profit les ressources matérielles, culturelles et intellectuelles à leur disposition en Europe. Précisons ici que l'intensité des attentes ne doit pas être confondue avec les formes que prend le suivi scolaire. L'investissement scolaire des parents n'est pas le fidèle reflet de l'importance qu'ils accordent à l'école. Cet investissement différencié renvoie avant tout aux inégalités d'aptitude et de ressource.

En fait, comme l'a bien montré Lahire (1995), l'histoire familiale et les trajectoires scolaires sont imbriquées en raison de la préexistence d'un synopsis fait d'espoirs parentaux et de contraintes socioéconomiques (Zéroulou, 1988). Dans la plupart des familles rencontrées, l'échec scolaire et les turbulences disciplinaires qui les accompagnent ne sont pas seulement des déceptions, mais bien souvent des drames, voire des trahisons. Des situations dont la portée dépasse la seule question du futur professionnel des enfants remuants.

En effet, l'ensemble des enjeux sociaux et symboliques que nous venons de présenter participe d'une très forte incrimination familiale de l'échec. Même si l'analyse parentale de ces situations peut mobiliser des arguments biographiques (leur propre incompétence), sociétaux (inégalités sociales et ghettoïsation) ou plus institutionnels (critique des établissements scolaires et des équipes éducatives), c'est la responsabilité individuelle des enfants qui est retenue en priorité. Incrimination familiale également repérée par Millet et Thin (2005) qui soulignent la condamnation des collégiens turbulents «au sein de l'univers familial pour leurs différents manquements scolaires et les désordres familiaux qu'ils occasionnent» (Millet et Thin, 2005, p. 67). Pour Sayad (1999), le fait que la plupart des immigrés algériens vivant en France - selon nous, ce raisonnement ne vaut pas que pour les migrants algériens n'ont pas eu accès à l'école fait qu'ils perçoivent leurs enfants comme les principaux ressorts de la revanche sociale. L'extrait d'entretien suivant contient les mots d'une mère de famille, immigrée malienne et 
dévoile la prégnance des mythes parentaux et des scénarios intimes qui en découlent:

- Mme D. (48 ans, 4 enfants, femme de ménage à temps plein): «Les choses elles changent, Marwan, quand on est arrivées au début en France et qu'on a eu des enfants, on était rêveuses. Surtout les mères africaines, tu sais elles parlent pas, mais au village on les a mariées pour la plupart. (...) Alors je te dis on est avec les premiers enfants très motivées, rêveuses. «Inch Allah, mon fils il sera ça, ma fille elle sera ça», comme ça ils nous aident, ils auront une bonne position ici en France et tout ça. (...) Au village, y avait la fille de l'épicier qui était secrétaire à la ville, elle était belle, très gentille, elle faisait les lettres de tout le monde, elle avait la machine à écrire. À l'époque pas d'ordinateur, c'était une grosse machine. Moi, c'était mon rêve ça. (...) Et mon fils, aujourd'hui, il lui manque rien, (...) j'attendais que ça, les études, quelque chose de bien. (...) Quand ils étaient petits, je rêvais qu'ils soient médecins, ingénieurs, des grands métiers, des «lumières» avec de l'argent et tout. C'est pour ça tu vois on n'a pas beaucoup d'enfants. Aujourd'hui, je veux juste ne pas avoir de problèmes avec la justice et la prison à éviter, c'est tout. C'est triste et ça me rend malheureuse mais c'est comme ça et je vis avec la peur là (elle montre son ventre).»

Les mythes auxquels s'attache Mme D. (l'image de la fille de l'épicier lettrée et rayonnante) et qu'elle appelle «rêve» s'inscrivent dans sa biographie, dans son parcours migratoire et surtout, ils circulent et sont partagés avec d'autres parents. La référence aux contrariétés du mariage, à l'absence de scolarisation et à la figure de la «fille de l'épicier» «belle» et surtout lettrée, souligne le caractère intime des attentes qui pèsent sur les enfants ainsi que leur antériorité. Bien évidemment, les attentes des mères et des pères peuvent diverger dans leur contenu, leur intensité, ou en fonction des enfants selon leur sexe et leur rang dans la fratrie (Mauger, 2003). Ces variations peuvent parfois - et non systématiquement - prendre appui sur des ressorts socioculturels (selon le rang social ou l'origine).

\section{L'abjuration parentale et ses effets}

Toutefois, le schéma de pensée dominant fait de la réussite à l'école l'un des critères essentiels d'accès à la reconnaissance parentale. L'échec en 
revanche ainsi que les comportements transgressifs sont source de déclassement familial et égratignent le statut personnel du déviant. Les enquêtés décrivent chacun à leur manière ces logiques et ces moments de réévaluation de leur statut personnel en famille et aux yeux de leurs parents, en fonction de leur scolarité et de leur attitude. Ils racontent ces moments de franchissement symbolique, notamment le passage entre dilection filiale et reniement symbolique. Ainsi, la qualité des relations entre les parents et chacun de leurs enfants repose sur leur degré de conformité par rapport aux mythes parentaux, aux attentes scolaires et aux normes établies. Il faut par ailleurs retenir que les changements de statut d'un membre de la fratrie ont tendance à créer un mouvement et des recompositions plus larges des affinités filiales. Cette disqualification intrafamiliale a des conséquences sur l'ordre éducatif ainsi que sur les destins juvéniles. À l'instar des «effets d'élection» qui découlent des «manières d'inciter l'enfant à "réussir" ou à travailler pour "réussir", selon les capacités familiales à aider l'enfant à réaliser les objectifs qu'on lui fixe» (Lahire, 1995, p. 27), «l'abjuration» parentale produit ses propres effets, reposant sur le même mécanisme d'autoréalisation.

Cette abjuration se manifeste dans divers registres pour ceux qui la subissent: transformation de l'humeur parentale, mise sous pression permanente et «tolérance zéro» face aux moindres écarts, disputes et reproches fréquents, etc. Certains parents ignorent les enfants qui les ont «trahis», à travers une ostensible mise au ban. En l'absence d'un retour à la normalité - ce qui est rarement le cas -, le temps et le sentiment d'impuissance parentale introduisent inexorablement un détachement réciproque que chaque partie envisage comme la voie la plus raisonnable. L'extrait d'entretien suivant illustre nos analyses en introduisant la dimension de seuil et l'idée d'étape dans les formes de réactions parentales.

- Lucien (22 ans, sans emploi): «(...) Avant de me boycotter totalement, ils (parents) ont d'abord essayé de me corriger avec des coups de pression, du... pour moi c'était du harcèlement quoi, "fais pas ci, fais pas ça, t'étais où? avec qui ?..." (...) C'est bien après que... en voyant qu'ils pouvaient pas grand-chose pour changer les choses dans leur sens, qu'ils ont commencé à faire comme si j'étais pas là, même ma dareune (mère) j'l'ai blasé carrément, elle faisait le... le service minimum pour moi.» 
Labjuration parentale et le déclassement familial produisent rarement les effets souhaités par les ascendants et se résument à une redistribution du déshonneur vécu. La dureté du traitement réservé au «paria» vise autant à sanctionner qu'à prévenir les turbulences éventuelles des puînés. Les enquêtés partagent l'idée qu'une réaction parentale est nécessaire mais que l'abjuration est excessive et institue durablement des inégalités dans la fratrie. Les effets observés sont tout autres. Le premier est de réduire la présence domestique du «paria» pour qui l'ambiance devient détestable. L'ambiance familiale est une expérience sensorielle, relationnelle et matérielle. C'est-à-dire une réalité phénoménologique formée d'un «ensemble de sensations, éprouvées in situ, propres au moment et au lieu dans lequel on se trouve», et qui tend à «définir un rapport particulier à l'espace environnant et au temps, c'est-à-dire une façon singulière de se sentir conjointement dans son corps propre et dans le lieu dans lequel on se trouve» (Bittolo, 2007, p. 48) ${ }^{4}$. Elle interdit durablement l'expression domestique de leurs subjectivités alors que la bande apparaît comme une scène de réalisation de soi. La concurrence qui oppose ces deux lieux de vie se joue notamment à travers la chaleur qu'ils dégagent, la publicité qu'ils offrent aux égos, la «débanalisation» de la vie quotidienne qu'ils permettent (Wacquant, 1995). Comparativement à l'inconfort que génère l'expérience domestique pour les enfants qui «posent problème», les bandes contraignent mais n'écrasent pas les subjectivités. Il n'est donc pas rare d'observer des jeunes expressifs dehors, se transformer dedans en adolescents brimés, nerveux et introvertis.

\section{Du déclassement des enfants à la délégitimation des parents}

Si à présent on privilégie le point de vue des enquêtés sur leurs parents, la dialectique attentes-attitudes permet d'analyser d'autres enjeux tout aussi décisifs. On se focalisera ici sur la question de l'autorité éducative qui, de manière assez surprenante, est rarement définie et interrogée comme un mécanisme en soi. Pourtant «l'autorité» est omniprésente dans les discours politiques, ceux des magistrats et des policiers, et des nombreux intervenants socioéducatifs. En France, la plupart des dispo-

4. Sur la dialectique climat familial et participation aux gangs, voir Cummings et Monti (1993); Maxson, Whitlock et Klein (1998); Vigil (1993). 
sitifs visant les parents de mineurs délinquants visent à «restaurer» l'autorité. Dans un sondage IFOP sur la situation dans les banlieues (février 2008), $42 \%$ des répondants jugeaient que la «démission des parents» expliquait le mieux les violences juvéniles. Les jeunes en bandes soumettent leurs parents à l'indignité publique, à la réprobation $\mathrm{du}$ voisinage ainsi qu'aux intrusions des institutions sociales, scolaires, municipales ou pénales. Et pourtant ces derniers s'en accommodent, même s'ils culpabilisent et qu'ils rechignent très souvent à en parler. Sur quoi repose cette acceptation tacite, quoique inassumée, des coûts payés par les parents.

\section{L'autorité éducative est symbolique}

La pensée politique oppose traditionnellement deux formes d'autorité, le potestas et l'auctoritas. Le potestas renvoie à des formes statutaire, établie, institutionnelle de pouvoir et d'autorité. À l'inverse, le pouvoir issu de l'auctoritas repose sur la crédibilité, c'est-à-dire une soumission librement acceptée. On retrouve cette opposition concernant l'autorité éducative dont les fondements sont bouleversés par les transformations de la famille contemporaine (de Munck, 2000; Théry, 1998). L'avènement de la psychologie et de l'adolescence comme âge de la vie ont favorisé l'émergence de nouvelles normes éducatives plaçant l'enfant au centre des attentions, dans un esprit de dialogue et d'écoute de leurs aspirations et subjectivités, et promouvant la négociation (Singly, 1996). Des normes promues en opposition aux pédagogies verticales, statutaires et disciplinaires, dans lesquelles les rôles de chacun sont adossés à la position familiale. Ces schémas familiaux fortement sexués, prégnants en bas de la hiérarchie sociale, ont perdu de leur légitimité, mettant en porte à faux ceux qui y restent attachés. L'une des transformations majeures se trouve dans l'obligation pour les parents de faire reposer leur autorité éducative sur leurs ressources personnelles. Mais avant d'aller plus loin, précisons ce que nous entendons par autorité éducative.

Les penseurs de l'autorité se rejoignent sur le fait que l'autorité et sa reconnaissance ne font qu'un (Kojève, 2004). Elle n'est ni manipulation, intimidation, contrainte physique, séduction, ni, ajoute Arendt (1995), «persuasion par arguments». Cela implique une asymétrie des positions entre la cible et la source de l'autorité. L'autorité éducative repose ainsi sur la crédibilité, le charisme, les compétences, composant un ensemble 
singulier de ressources suscitant ce que Weber (1995, p. 219) appelle la "volonté d'obéir», c'est-à-dire la légitimité comme forme de dominance symbolique. Pour le psychiatre Mendel (2002, p. 33), «l'autorité (...) nécessite un additif pour que l'individu puisse revendiquer une sphère d'influence plus large (...). Cet additif, c'est la légitimité». Par quoi est composé cet «additif»? Les théoriciens divergent à ce sujet tant les ressorts individuels et culturels de légitimation sont nombreux. Pour eux, la légitimité est une forme de ressource symbolique reposant sur l'antériorité, l'image publique (Revault d'Allones, 2006), le capital social et la reconnaissance des compétences de vie. Jugés légitimes, donc crédibles, les parents pourront alors déployer leurs valeurs, leurs normes pour que leurs enfants en fassent des intuitions, une conscience, des repères solides puis des choix de vie, ou du moins une puissante source d'inspiration. La dialectique légitimité-délégitimation est fondamentalement une affaire de schèmes de perception et de système d'attente. De la même manière que les attentes et les mythes parentaux forgent une image de l'enfant souhaité, espéré, rêvé, etc., les attentes de leurs enfants, sensibles aux modèles dominants, voire publicitaires, d'épanouissement familial, façonnent une image de la parentalité souhaitée. Celle-ci constitue une grille de jugement moral et symbolique des parents réels, un filtre à travers lequel se joue leur crédibilité éducative. La délégitimation éducative rend visibles ces dissonances entre familles idéalisées et familles vécues:

Note du 08/03/2009: Sollicité par madame Gh. (femme au foyer, mari peintre en bâtiment intérimaire, 5 enfants, appartement de 3 pièces) pour les problèmes disciplinaires de son fils cadet Yann (18 ans, BEP Vente, fort absentéisme), j'assiste à plusieurs disputes familiales et à un incident entre les parents. Ces disputes concernent le passage imminent de Yann en conseil de discipline (d'où sera prononcée son exclusion définitive). Ce dernier, gêné par ma présence, a tenté de faire redescendre la tension entre ses parents qui, emportés par la colère, m'ont progressivement oublié. Les propos du père étaient décousus et il avait parfois du mal à contrôler ses émotions, tandis que la mère tentait de minimiser les risques de renvoi définitif. Une fois le calme revenu, j'ai pu lire la honte dans les yeux de Yann, pendant que sa mère s'excusait et que son père continuait de manifester son mécontentement et de déployer son argumentaire dans un étrange monologue. Lors de mon départ, Yann a souhaité faire un bout de chemin avec moi. Après avoir exprimé sa gêne pour les incidents, il a eu des mots assez durs sur ses 
parents et le fonctionnement de sa famille qu'il opposait à une «famille normale» dans laquelle «les gens s'entendent, s'expliquent tranquillement».

L'expression «famille normale» exprime l'intériorisation des normes dominantes à travers l'action des institutions appuyée par celle des médias (émissions de télévision, publicité, fictions), mais également la fréquentation de foyers où «les gens se parlent», «s'entendent», «se respectent», «font des choses ensemble» ou les «parents passent bien» et «s'intéressent à toi». Bien entendu, les ingrédients d'une «famille normale» ou de parents idéaux varient d'un jeune à l'autre, mais ils se réfèrent tous au schéma majoritaire. L'idéal familial et le disponible sont ainsi l'objet de jugement et de classement, de validation, de critique ou de rejet des postures parentales surannées. Comme le soulignait Percheron (1985), des obstacles éducatifs supplémentaires se dressent lorsque les modèles transmis ou incarnés par les parents ne sont pas relayés par la société. Le désaveu, même implicite, des institutions dominantes entrave considérablement les capacités de transmission des parents tout en déstabilisant et tiraillant leurs enfants, attirés par les schémas que promeuvent des groupes sociaux qui stigmatisent leurs ascendants. Cette dissonance entre parentalité souhaitée et parentalité vécue est au cœur de l'analyse des échecs éducatifs, en permettant de décrire et d'analyser le processus de disqualification qui affecte les parents. La délégitimation éducative est donc un déplacement - dont la durée et la profondeur sont variables - des systèmes de référence et d'autorité de la famille vers la rue, des éducateurs vers la bande, qui implique une commutation des compétences normatives. C'est un processus lent, progressif, dont nous avons repéré deux formes typiques qui, très souvent, se combinent et s'articulent.

\section{Délégitimation commisérative et délégitimation morale}

La délégitimation commisérative, la plus fréquemment rencontrée, repose sur la dépréciation du statut symbolique des parents qui érode leur crédibilité éducative, leur pouvoir de figuration autoritaire, leur capacité à dire le monde et à s'y repérer. Les jeunes qui s'y réfèrent ont recours à des arguments pointant le déterminisme et la fatalité sociale. Elle repose sur la disqualification sociale des parents, c'est-à-dire sur l'infériorisation de leur statut et de tout ou partie de leur image publique. 
Comme précédemment énoncé, l'autorité éducative repose sur un principe de dominance symbolique, une forme de capital social qui s'apparente sans s'y confondre à la notion grecque de charisma qui signifie la "grâce» attachée à une personne:

- Enquêteur: «On peut dire que tes parents te servent de modèle? Des personnes qui t'inspirent?»

- Fathi (22 ans, en formation cariste): «Modèle? Franchement non, c'est pas mes modèles, j'vais pas te mentir. M'inspirer?... Franchement non plus, m'inspirer quoi? Perso, mes parents ils se cassent le cul pour nous, mon daron (père) fait un taf (travail - il est agent de nettoyage) de merde, faut dire c'qui y est, et ça on le respecte, voilà j'respecte les... efforts voilà, les efforts, la patience et tout. (...) Mais m'inspirer... Ceux qui m'inspirent, ils maîtrisent le truc, tu vois, ils maîtrisent quelque chose, tu sais qu'ils ont fait leurs preuves. Mais nos darons, franchement, ils sont à l'ouest (décalés, en difficulté), ils maîtrisent rien.»

Dans la bouche de Fathi, très actif dans sa bande depuis qu'il a 14 ans, l'idée de charisma que l'on a introduite avec la notion de "modèle» repose sur la «maîtrise» et le fait de faire ses «preuves». Les enquêtés ${ }^{5}$ associent des compétences et des expériences différentes à l'idée de maîtrise, même si les dimensions du pouvoir, de la réussite matérielle et de la place dans la hiérarchie sociale reviennent régulièrement. De ce point de vue, la délégitimation commisérative apparaît comme une contrainte sociale qui pèse particulièrement, pour ne pas dire spécifiquement, sur les parents qui appartiennent aux groupes sociaux dominés et les plus exposés au stigmate. Les parents que nous avons rencontrés vivent dans l'inconfort économique et font face à des problèmes récurrents qui les amènent à solliciter de l'aide. Même les qualités que leur reconnaissent leurs enfants sont souvent indissociables de leurs épreuves. Pour Fathi, ses parents «se cassent le cul» et méritent donc le respect pour leurs «efforts, la patience et tout». D'autres diront de leurs parents qu'ils «galèrent», qu'ils «rament» et qu'ils «font ce qu'ils peuvent». Tout se passe comme si l'identité sociale des ascendants était indissociable de leurs difficultés, laissant dans l'ombre, par un jeu de sélection, d'autres aspects de leur personnalité. Il nous semble que

5. Précisons que ces regards dépréciatifs sont personnels: au sein d'une même fratrie, différents regards sur les parents peuvent cohabiter, s'opposer, voire se confronter. 
la délégitimation est facilitée par la culture des bandes qui promeut la puissance personnelle, économique ou la virilité comme pouvoir. Dans le quartier de l'enquête, le prestige social que valide l'expression «être en place» repose plus largement sur la capacité d'agir sur sa vie, de s'ouvrir des voies et d'échapper aux lois du déterminisme. Des normes qui survalorisent le pouvoir personnel et l'image publique et participent d'une hiérarchisation des qualités sociales défavorable à leurs parents.

Dans ces classements, le statut socioprofessionnel occupe une place prédominante, tout comme la position locale à partir d'une distinction établis/marginaux (Elias et Scotson, 1997) ou les dissonances culturelles. La prédominance aux yeux des enquêtés du statut social traduit sa centralité dans le contexte français. Nous avons également noté leur insistance sur les difficultés langagières, le style vestimentaire, puis ce qui relève de l'alcoolisme ou de la santé mentale. Des caractéristiques - un jeune a employé le mot «défaut»-dont l'importance semble croître à mesure que le conflit s'installe en famille. La tonalité des récits n'est pas sans rappeler les témoignages de deux transfuges de classe tels Ernaux (1997) ou de Eribon (2013) sur la honte qu'ils éprouvaient à l'égard de leurs parents. Une honte qui n'est pas source de reproches à leur égard, mais qui invite à s'en détourner sans les condamner, à les protéger tout en les mettant à distance, à assumer ensemble affection et disqualification. Whyte (2002, p. 38) soulignait déjà que «les enfants sont souvent fort attachés à leurs parents et pourtant ils éprouvent une certaine condescendance à leur égard». "Condescendance» en partie forgée et régulièrement renouvelée par l'intervention des institutions. Celles-ci jouent un rôle déterminant dans la délégitimation commisérative à travers leur capacité à dire la norme, à désigner, à classer et donc à évaluer les individus. Si l'hostilité des jeunes en bandes à l'égard des institutions est régulièrement soulignée par la recherche, ses sources sont plus rarement étayées. Or, le traitement des parents par les institutions influence en profondeur les postures des enfants à leur égard. Le stigmate "jette un discrédit profond sur celui qui le porte» (Goffman, 1975, p. 13), notamment dans une société dans laquelle l'image publique et le prestige jouent un rôle moteur (Honneth, 2000). Ainsi, les adolescents sont autant liés à l'image publique de leurs parents que ces derniers aux discours publics qui les désignent:

- Serge (19 ans, sans emploi) : «(...) Tu veux que je pense quoi de mon daron quand il fait le lion à la maison et qu'il se transforme en poule dès qu'on entre dans le bureau d'une juge?» 
- Krimo (21 ans, sans emploi): «(...) Ça c'est un truc que je supporte pas, ça... quand on est convoqués, que le proviseur il fait pas la différence entre mon daron et moi... si, moi, il me tutoie, lui, il lui dit vous... mais le ton, la manière de parler, de hagar (d'humilier), c'est la même. Mon daron, il se plie en quatre «oui monsieur, oui monsieur», mais moi, je suis za'af (énervé) avec une force! J'ai envie de défoncer le proviseur et j'ai une rage contre mon daron...»

C'est dans le cadre disciplinaire à l'école ou au sein des instances pénales que le stigmate des parents est le plus fréquemment dénoncé par les enfants, notamment en raison de la violence de son expression. Dans des registres moins rugueux, le travail social participe parfois du même processus de disqualification, des mêmes «rituels de dégradation» (Garfinkel, 1956), en raison d'interventions qui infantilisent et actualisent publiquement la situation de dépendance des parents (Paugam, 1991). Des interactions qui prolongent les nombreux discours dépréciatifs tenus dans la sphère publique à l'égard des pauvres, des habitants des quartiers populaires, des minorités et religions impopulaires (Hajjat et Mohammed, 2013; Mucchielli, 2001) dont sont parfois issus les enquêtés.

Le second processus de délégitimation que nous avons repéré diffère du premier en ce qu'il ne met pas en jeu la position sociale mais le statut moral des parents. Si dans la logique de commisération, leur «décote sociale» ne leur est pas imputable, car située dans l'ordre des choses («c'est comme ça») (Bourdieu, 1993), la délégitimation morale est, aux yeux des enquêtés, le résultat de choix qui révèlent probité et personnalité. Elle vise des attitudes qui s'opposent aux valeurs et aux normes que les enfants souhaiteraient voir structurer les attitudes parentales.

En fréquence, la délégitimation morale s'exprime moins fréquemment que la délégitimation commisérative $e^{6}$ et vise davantage les pères que les mères. Elle concerne essentiellement des attitudes d'une certaine gravité se distinguant d'une conflictualité routinière dans les familles, comme celles qui portent sur la négociation de l'ordre domestique. La délégitimation morale procède globalement de deux temporalités distinctes déjà repérées par Monod (1968, p. 308) à l'époque des blousons noirs: «on peut donc envisager deux types de situations négatives: celle où la

6. On peut à l'inverse penser qu'elle est plus fréquente dans les milieux sociaux moins exposés au stigmate. 
rupture avec la famille est consommée, celle où le désaccord mûrit progressivement, sans aboutir nécessairement à la rupture». La seconde temporalité, la plus fréquente, se déploie à l'adolescence, lorsque la détérioration scolaire assombrit les destins sociaux et surtout lorsque les parents ne peuvent plus bénéficier de la modération psychologique de l'enfance.

Les raisons invoquées varient selon les enquêtés, certaines visant des situations récurrentes, d'autres des ruptures et des déceptions soudaines et profondes. La qualité du climat familial sert souvent de cadre de jugement lorsque les enquêtés dénoncent des formes récurrentes d'antipathie parentale, de froideur, de distance, d'aigreur ou de rouspétance:

- Marvin (18 ans, apprenti plombier): «perso j'en peux plus d'entendre mon daron (père) râler «h24» (toute la journée) pour un oui ou pour un non. T'as l'impression qu'il est programmé pour ça... il rend fou (...). Quand t'es petit, tu t'y fais, c'est le daron quand même avec toute l'image du daron. Mais ça fait quelques années, il rend fou, il... met la pression non stop, c'est du... comment ça s'appelle le truc?... du harcèlement, voilà du harcèlement. (...) ça te fout une ambiance de merde à la maison où tout le monde cherche la petite bête... (...) Ce qui me fout le plus la rage par rapport à mon père surtout, c'est que son délire a pété pour de bon nos relations à la maison».

Les sources sociales et familiales de la rouspétance et de l'antipathie chroniques du père influencent peu les colères et l'inimitié de Marvin. Ce dernier n'a qu'une vague connaissance de l'histoire de ses parents, de leurs douleurs intimes souvent tues. Il y a peu d'échanges dans cette famille dominée par des ressentiments amplifiés par l'appartenance active de Marvin à une bande locale. D'autres contentieux assez récurrents renvoient aux inégalités de traitement entre les membres de la fratrie. Comme l'avait souligné Kojève (2004), «il y a des conflits entre l'obéissance filiale et le sentiment de justice» (p. 77). L'équité parentale est une norme prégnante pour les enquêtés qu'insupportent l'usage fréquent de comparaisons souvent vexantes, une distribution inégale de l'affection, de l'attention ou des ressources matérielles du foyer. Toutefois, il est difficile de distinguer ce qui précède de ce qui résulte des démobilisations scolaires et des conduites délinquantes. Si très souvent le déclassement familial des enfants découle des déceptions parentales, il arrive parfois qu'il précède la dégradation des scolarités et l'émergence des inconduites. 
La délégitimation morale se noue dans de nombreuses autres circonstances qu'il n'est guère possible d'énumérer ici. L'usage systématique de châtiments corporels, la violence conjugale, la dépendance aux psychotropes ou aux jeux d'argent, la «méchanceté» ou le «vice», l'égoïsme, etc., constituent autant de schémas de déprise éducative dont les manifestations, sans absoudre moralement les parents, sont fortement influencées par leur appartenance sociale et leurs conditions d'existence:

- Jabar (17 ans, sans emploi): «(...) tout ce que je t'ai dit, je l'ai dit parce que c'est connu. Ma daronne, elle fout sa merde partout avec sa grande gueule de commère et mon daron, il est blacklisté au PMU de la gare. Après, y a d'autres trucs que j'te dis qu'ils existent mais c'est impossible que je dise quoi. C'est trop dur en fait. (...) Mais la conséquence c'est que j'ai une rage de malade contre les deux. (...) Je sais tu vas me dire le respect des parents tout ça... ça c'est ok quand tes darons (parents), ils sont respectables. Moi c'est pas des parents, c'est des serpillières ».

Le souci de ne pas amplifier la stigmatisation des parents de milieu modeste l'emporte parfois sur les épreuves qu'ils s'imposent et qu'ils imposent à leurs enfants. Sur ce point, les injonctions visant à sacraliser le statut moral des parents irritent très souvent les jeunes comme Jabar qui éprouvent colère et ressentiment à leurs égards et qui estiment qu'ils s'autocensurent trop au nom de la dignité familiale. La description des réalités parentales est coûteuse nerveusement car la mise en accusation des ascendants est une épreuve, y compris pour ceux qui tiennent les propos les plus violents en prenant un air détaché ou qui donne l'impression d'en jouer. Si les enquêtés portent un jugement moral sur les pratiques de leurs parents, et réciproquement, c'est qu'à leurs yeux, il existe un champ des possibles mal ou pas exploité, contrairement à la délégitimation commisérative qui s'impose par la verticalité des rapports de domination et la puissance du stigmate social.

\section{Conclusion}

Pas d'éducation sans rayonnement, pas d'ascendant sans légitimité. Les deux logiques de disqualification présentées ici invitent à saisir empiriquement la mécanique psychosociale de l'autorité et de l'action éducative. Ces formes de dépréciation ne sont jamais absolues, elles varient en fonction des configurations relationnelles, de la situation sociale ainsi 
que de la trajectoire morale des familles (Mohammed, 2011). Qu'il s'agisse de comprendre la formation des bandes ou bien d'agir dessus, la prise en compte de ces logiques de dépréciation symbolique est inévitable tant elles influencent la relation éducative et le climat familial. Ces représentations agissent comme des filtres actifs dans la relation quotidienne. Ce niveau spécifique de la relation filiale doit évidemment être articulé aux autres facteurs familiaux de la délinquance pour adopter la vision la plus globale possible, il invite également à réinterroger leur portée heuristique et théorique. Pour illustrer ce constat, prenons l'exemple de la supervision parentale. Elle est, selon LeBlanc (1988), la variable clé des dynamiques familiales, dont le niveau de corrélation statistique avec la délinquance juvénile est le plus élevé. Or, cette variable est souvent étudiée sans être liée à ses conditions symboliques et matérielles de réalisation. Que peut valoir une pratique intensive de supervision pour des parents délégitimés? Son efficacité ne repose-t-elle pas nécessairement sur l'autorité éducative, c'est-à-dire sur la capacité pour les parents ou tout autre éducateur d'imposer la norme et de fixer un cap? Par ailleurs, s'agissant des dispositifs publics d'intervention, que peuvent prétendre des dispositifs institutionnels qui se désintéressent des ressorts symboliques des difficultés éducatives des parents? Leur perte d'autorité n'est-elle pas aggravée par des pratiques, des paroles, des regards professionnels qui creusent leur déficit symbolique tout en prétendant «restaurer leur autorité»? Au final, la connaissance gagnerait à réduire un tel déséquilibre entre la captation statistique et l'observation ancrée du détachement entre les jeunes en bande et leurs parents.

\section{Références}

Arendt, H. (1995). La crise de la culture. Paris, France: Gallimard.

Bibeau, G. et Perreault, M. (2003). La gang: une chimère à apprivoiser: marginalité et transnationalité chez les jeunes d'origine afro-antillaise. Montréal, Québec: Les Éditions du Boréal.

Bittolo, C. (2007). Ambiances et changements en analyse de groupe. Connexions, $88(2), 9-62$.

Boucher, M. (2011). Gouverner les familles. Les classes populaires à l'épreuve de la parentalité. Paris, France: L'Harmattan.

Bourdieu, P. (1993). L'ordre des choses. Dans P. Bourdieu (dir.), La misère du Monde (p. 12-17). Paris, France: Seuil.

Cernkovich, S. A. et Giordano, P. C. (1987). Family relationships and delinquency. Criminology, 25(2), p. 295-319. 
Cummings, S. et Monti, D. J. (1993). Gangs: The Origins and Impact of Contemporary Youth Gangs in the United States. Albany: State University of New York Press.

De Munck, J. (2000). Les métamorphoses de l'autorité. Dans Collectif d'auteurs (dir.), Quelle autorité? Une figure à géométrie variable (p. 21-42). Paris, France: Autrement.

Elias, N. et Scotson J. L. (1997). Logiques d'exclusion. Enquête sociologique au cœur des problèmes d'une communauté. Paris, France: Fayard.

Eribon, D. (2013). La société comme verdict. Paris, France: Fayard.

Ernaux, A. (1997). La Honte. Paris, France: Gallimard.

Garfinkel, H. (1956). Conditions of Successfull Degradation Ceremonies. American Journal of Sociology, 61, 420-424.

Gautron, V. (2014). Le positionnement des travailleurs sociaux dans les dispositifs locaux de coproduction de la sécurité. Dans D. Attias et L. Khaiat (dir.), Enfants rebelles, parents coupables? (p. 115-130). Toulouse, France: Eres.

Goffman, E. (1975). Stigmate. Les usages sociaux des handicaps. Paris, France: Éditions de Minuit.

Gove, W. R. et Crutchfield, R. D. (1982). The Family and Juvenile Delinquency. The Sociological Quarterly, 23(3), 301-319.

Hajjat, A. et Mohammed, M. (2013). Islamophobie. Comment les élites françaises fabriquent le "problème musulman». Paris, France: La Découverte.

Hamel, S. et Brisebois, R.-A. (2011). Regard intime sur le phénomène des gangs. Des parents racontent leur histoire: parcours migratoires inachevés et pertes de repères identitaires. Dans M.-L. Martinez (dir.), Adolescents délinquants est leurs parents. Bandes et violences en groupe (p. 57-95). Paris, France: L'Harmattan.

Hébert, J., Hamel, S., Savoie, G., Fredette, C., Blais, M.-F. et Bertot, J. (1997). Jeunesse et gangs de rue (Rapport de recherche). Montréal, Québec: Institut de recherche pour le développement social des jeunes.

Hirschi, T. (1969). Causes of Delinquency. Berkeley, CA : University of California Press.

Honneth, A. (2000). La lutte pour la reconnaissance. Paris, France: Éditions du cerf.

Jaggers, J., Church, W. T., Tomek, S., Bolland, K. A., Hooper, L. A et Bolland J. (2013). Predictors of gang involvement: a longitudinal analysis of data from the mobile youth Survey. Journal of the Society for Social Work and Research, 4(3), 277-291.

Kojève, A. (2004). La notion de l'autorité. Paris, France: Gallimard.

Lahire, B. (1995). Tableaux de familles. Heurs et malheurs scolaires en milieux populaires. Paris, France: Gallimard/Seuil.

Leblanc, M. (1988). Des années 1970 aux années 1980: changements sociaux et rôle de la famille dans l'explication de la conduite délinquante des adolescents. Annales de Vaucresson, 28(1), 159-187.

Mauger, G. (2003). Le monde des bandes et ses transformations. Une enquête ethnographique dans une cité HLM (Rapport final). Paris, France: DIV. 
Maxson, C. L., Whitlock, M. L. et Klein, M. W. (1998). Vulnerability to street gang membership: Implications for practice. Social Service Review, 72, 70-91.

Mendel, G. (2002). Une histoire de l'autorité. Permanences et variations. Paris, France: La Découverte et Syros.

Millet, M. et Thin, D. (2005). Ruptures scolaires. L'école à l'épreuve de la question sociale. Paris, France: PUF.

Mohammed, M. (2011). La formation des bandes. Entre la famille, l'école et la rue. Paris, France: PUF.

Monod, J. (1968). Les Barjots. Paris, France: Julliard.

Mucchielli, L. (2000). "Familles et Délinquance», Dossiers d'études no 9. Paris, France: CNAF.

Mucchielli, L. (2001). Violences et insécurité. Fantasmes et réalités dans le débat français. Paris, France: La Découverte.

Observatoire national des zones urbaines sensibles [ONZUS]. (2005). Rapport 2005. Paris, France: auteur.

Percheron, A. (1985). Le domestique et le politique. Types de familles, modèles d'éducation et transmission des systèmes de normes et d'attitudes entre parents et enfants. Revue française de science politique, 35(5), 840-891.

Poullaouec, T. (2004). Les familles ouvrières face au devenir de leurs enfants. Économie et Statistique, 371, 3-22.

Rankinand, J. H. et Wells, L. E. (1990). The effects of parental attachments and direct controls on delinquency. Journal of Research in Crime and Delinquency, 27, 40-65.

Revault d'Allones, M. (2006). Le pouvoir des commencements: essai sur l'autorité. Paris, France: Seuil.

Sayad, A. (1999). La double absence. Des illusions de l'émigré aux souffrances de l'immigré. Paris, France: Seuil.

Singly, F. (2002). Le soi, le couple et la famille. Paris, France: Nathan.

Smith, C. A. et Stern, S. B. (1997). Delinquency and antisocial behavior: a review of family processes and intervention research. Social Service Review, $71(3), 382-420$.

Svensson, R. (2004). Shame as a Consequence of the Parent-Child Relationship: A Study of Gender Differences in Juvenile Delinquency. European Journal of Criminology, 1(4), 477-504.

Théry, I. (1998). Couple, filiation et parenté aujourd'bui (Rapport à la ministre de 1'Emploi et de la Solidarité et au Garde des Sceaux). Paris, France: Ministère de la Justice.

Vallet, L.-A. et Caille, J.-P. (2000). La scolarité des enfants d'immigrés. Dans A. Van Zanten (dir.), L'école: l'état des savoirs (p. 293-301). Paris, France: La Découverte.

Van de Velde, C. (2008). Devenir adulte. Sociologie comparée de la jeunesse en Europe. Paris, France: PUF.

Vigil, J. D. (1993). The Established Gang. Dans S. Cummings et D. J. Monti (dir.), Gangs: the origins and impact of contemporary youth gangs in the United States (p. 95-112). New York, NY: State University of New York Press. 
Wacquant, L. (1995). Protection discipline et honneur: une salle de boxe dans le ghetto américain. Sociologie et sociétés, 27(1), 75-90.

Weber, M. (1995). Économie et société. Paris, France: Plon.

Whyte, W. F. (2002). Street Corner Society. Paris, France: La Découverte.

Young, T., Fitzgibbon, W. et Silverstone, D. (2014). A Question of Family? Youth and Gangs. Youth Justice August, 14, 171-185.

Zeroulou, Z. (1988). La réussite scolaire des enfants d'immigrés. L’apport d'une approche en termes de mobilisation. Revue françdise de sociologie, 29(3), 447470.

\title{
The Gang Youths and Their Parents. Symbolic Patterns of Detachment
}

\begin{abstract}
Analysis of the role a family plays in youth gang formation involves something of a paradox. Intrafamilial functioning is commented on and deduced rather than directly observed, while the interventions of authorities are increasingly intrusive and constraining. One of the explanations for this contrast lies in methodology. Statistical approaches tend to hide the micro-sociological processes that are part of close family relationships and family symbolism. The challenge is to find a way to undertake studies that take into account the structure and symbolic aspect of the family. This article uses empirically based analysis to look at the reciprocal ideas and moral judgments of gang youths and their parents. The analyses suggests that it is necessary to question, or to change, the way researchers on juvenile delinquency investigate and analyse family factors relating to juvenile delinquency, particularly the phenomenon of youth gangs.
\end{abstract}

KEYWORDS - Delinquency, family, authority, youth gangs, education.

\section{Los jóvenes en banda y sus padres. Los caracteres simbólicos del desapego}

RESUMEN - El análisis del rol de las familias en la formación de bandas de jóvenes muestra un aspecto paradójico. El funcionamiento intra-familiar es más comentado, deducido, que directamente observado en situación. Paradoja que se ve reflejada en las intervenciones institucionales cada vez más intrusivas y apremiantes. Una de las explicaciones reside sin duda en la metodología utilizada. El predominio de los enfoques estadísticos tiende a ocultar los procesos micro-sociológicos anidados en los pliegues intimos de las relaciones y de la simbología familiar, que necesitan dispositivos de observación anclados. Ése es el objeto del presente artículo, que apunta a poner en perspectiva el peso de las representaciones y de los juicios morales recíprocos entre los jóvenes de una banda y sus padres. Los análisis proponen cuestionar e incluso renovar la manera en la que la investigación cuestiona y teoriza los factores familiares de la delincuencia juvenil, particularmente el fenómeno de las pandillas juveniles.

Palabras Clave - Delincuencia, familia, autoridad, bandas de jóvenes, educación. 